\title{
Effect of the Supporting Electrolyte Anion on the Thickness of PSS/PAH Multilayer Films and on Their Permeability to an Electroactive Probe
}

\author{
Alae E. El Haitami, ${ }^{\dagger, \$}$ David Martel, ${ }^{\S}$ Vincent Ball, ${ }^{\dagger,}$ Hoan Cong Nguyen, ${ }^{\S}$ Eric Gonthier, \\ Pierre Labbé, ${ }^{\perp}$ Jean-Claude Voegel, ${ }^{\dagger, *}$ Pierre Schaaf,,$"$ Bernard Senger, ${ }^{+\dagger, *}$ and \\ Fouzia Boulmedais ${ }^{\prime \prime}$ \\ Institut National de la Santé et de la Recherche Médicale, Unité 977, 11 rue Humann, \\ 67085 Strasbourg Cedex, France; Université de Strasbourg, Faculté de Chirurgie Dentaire, 1 Place de \\ l'Hôpital, 67000 Strasbourg, France; Laboratoire d'Electrochimie et de Chimie Physique du Corps Solide, \\ UMR 7177, CNRS/UdS, Institut de Chimie, 4, rue Blaise Pascal, 67070 Strasbourg Cedex, France; Centre \\ National de la Recherche Scientifique, UPR 22, Institut Charles Sadron, 23 rue du Loess, BP 84047, \\ 67034 Strasbourg Cedex 2, France; and Université Joseph Fourier Grenoble 1, CNRS, Département de \\ Chimie Moléculaire, UMR 5250, 38041 Grenoble Cedex 9, France
}

Received October 23, 2008. Revised Manuscript Received December 4, 2008

\begin{abstract}
Quartz crystal microbalance and cyclic voltammetry are used to investigate the influence of the supporting salt of polyelectrolyte solutions on the buildup and the structure of PSS/PAH polyelectrolyte multilayers (PSS: poly(4-styrene sulfonate); PAH: poly(allylamine hydrochloride)). This film constitutes a model polyelectrolyte multilayer system. The supporting electrolytes were sodium salts where the nature of the anion was changed by following the Hofmeister series from cosmotropic to chaotropic anions $\left(\mathrm{F}^{-}, \mathrm{Cl}^{-}, \mathrm{NO}_{3}{ }^{-}, \mathrm{ClO}_{4}{ }^{-}\right)$. For all the investigated anions, the film thickness increases linearly with the number of deposition steps. We find that chaotropic anions lead to larger thickness increments per bilayer during the film buildup than cosmotropic ones, confirming results found on PSS/PDADMA multilayers (PDADMA: poly(diallyldimethylammonium)). Films constituted by more than nine PSS/PAH bilayers are still permeable to hexacyanoferrate(II) ions, $\mathrm{Fe}(\mathrm{CN})_{6}{ }^{4-}$, whatever the nature of the supporting salt anion. On the other hand, these films are impermeable to ruthenium(II) hexamine ions, $\mathrm{Ru}\left(\mathrm{NH}_{3}\right)_{6}{ }^{2+}$, after the third PAH layer in the presence of $\mathrm{NaF}$, $\mathrm{NaCl}$, or $\mathrm{NaNO}_{3}$. These results are explained by the presence of an excess of positive charges in the film, which leads to a positive Donnan potential. We find that this potential is more positive when more chaotropic anions are used during the film buildup. We also find that a film constructed in the presence of chaotropic anions swells and becomes more permeable to $\mathrm{Fe}(\mathrm{CN})_{6}{ }^{4-}$ ions when the film is brought into contact with a solution containing more cosmotropic anions. All our experimental findings can be explained by a strong interaction between chaotropic anions with the $\mathrm{NH}_{3}{ }^{+}$groups of PAH that is equivalent, as far as the multilayer buildup and electrochemical response is concerned, to a deprotonation of PAH as it is observed when the film is constructed at a higher $\mathrm{pH}$. We thus arrive to a coherent explanation of the effect of the nature of the anions of the supporting electrolyte on the polyelectrolyte multilayer. We also find that great care must be taken when investigating polyelectrolyte multilayer films by electrochemical probing because electrochemical reactions involving the probes can appreciably modify the multilayer structure.
\end{abstract}

\section{Introduction}

The buildup of polyelectrolyte multilayer (PEM) films is influenced by various parameters such as the nature of the polyelectrolytes, the salt concentration ${ }^{1}$ and the $\mathrm{pH}$ of the polyelectrolyte solution ${ }^{2}$ and rinsing solutions, the exposure time, as well as the temperature. ${ }^{3,4}$ As observed in a large variety of physicochemical and biological processes, ${ }^{5}$ the nature of the salt, especially that of the salt anion, plays an important role in

* To whom correspondence should be addressed. Phone: +33-(0)390243258; Fax: +33-(0)390-243379; E-mail: bernard.senger@medecine.ustrasbg.fr.

" Institut National de la Santé et de la Recherche Médicale.

* Université de Strasbourg.

${ }^{\S}$ Laboratoire d'Electrochimie et de Chimie Physique du Corps Solide.

"Centre National de la Recherche Scientifique.

${ }^{\perp}$ Université Joseph Fourier Grenoble 1.

(1) Ladam, G.; Schaad, P.; Voegel, J.-C.; Schaaf, P.; Decher, G.; Cuisinier, F. J. G. Langmuir 2000, 16, 1249-1255.

(2) Yoo, D.; Shiratori, S. S.; Rubner, M. F. Macromolecules 1998, 31, 43094318.

(3) Tan, H. L.; McMurdo, M. J.; Pan, G.; Van Patten, P. G. Langmuir 2003, 19, 9311-9314.

(4) Salomäki, M.; Vinokurov, I. A.; Kankare, J. Langmuir 2005, 21, 1123211240.

(5) Cacace, M. G.; Landau, E. M.; Ramsden, J. J. Q. Rev. Biophys. 1997, 30, 241-277. the PEM buildup. The importance of both the salt concentration and the salt type on the PSS/PDADMA buildup (PSS: poly(4styrene sulfonate; PDADMA: poly(diallyldimethylammonium)) was already underscored by Dubas and Schlenoff. ${ }^{6}$ Von Klitzing et al. also stressed the difference between cosmotropic (relatively small polarizability) and chaotropic (higher polarizability) ions in the buildup of PEMs ${ }^{7}$ and Lo Nostro et al. emphasized the polarizability as a key factor of the anion specificity. ${ }^{8}$ Salomäki et al. have shown that not only the thickness of the multilayers obtained after a given number of alternated deposition steps of PSS and PDADMA depends significantly on the anion of the sodium salt used but even the evolution of the thickness with the number of deposition steps. ${ }^{9}$ The nature of the anions during the film buildup can also change the swelling of films as well as their mechanical properties. ${ }^{10,11}$ Whereas the evolution of the thickness is linear for sodium fluoride, it becomes exponential

(6) Dubas, S. T.; Schlenoff, J. B. Macromolecules 1999, 32, 8153-8160.

(7) von Klitzing, R.; Wong, J. E.; Jaeger, W.; Steitz, R. Curr. Opin. Colloid Interface Sci. 2004, 9, 158-162.

(8) Lo Nostro, P.; Lo Nostro, A.; Ninham, B. W.; Pesavento, G.; Fratoni, L.; Baglioni, P. Curr. Opin. Colloid Interface Sci. 2004, 9, 97-101.

(9) Salomäki, M.; Tervasmäki, P.; Areva, S.; Kankare, J. Langmuir 2004, 20, 3679-3683.

(10) Salomäki, M.; Kankare, J. Macromolecules 2008, 41, 4423-4428. 
for sodium bromide, and the film thickness for a given number of deposition steps follows the Hofmeister series where the monovalent anions are ranked according to their hydration entropy. ${ }^{9}$ It can however be emphasized that the origin of the Hofmeister effect is still not fully understood and the Hofmeister series should merely be taken as a phenomenological ranking. The thickness of the films was expected to be even higher and the thickness evolution even "more" exponential in the presence of $\mathrm{NaClO}_{4}, \mathrm{NaSCN}$, and $\mathrm{NaI}$ than with $\mathrm{NaBr}$, but PDADMA precipitated with these salts in the author's experimental conditions. The stronger increase of the film thickness with more chaotropic anions was explained by a stronger interaction between the polycation and these ions and thus more efficient screening of the polycation charges. ${ }^{9}$ This leads to polyelectrolyte deposition in more loopy conformations and thus to thicker films.

PSS/PAH (PAH: poly(allylamine hydrochloride)) constitutes another, widely used polyelectrolyte combination for the buildup of PEM films. It was the first system where the stratified nature of linearly growing multilayers was established. ${ }^{12}$ Whereas, for the PSS/PDADMA system, exponential growth of the film can be observed, it is only observed under very high ionic strength conditions for the PSS/PAH system indicating a stronger interaction between PSS and PAH than between PSS and PDADMA. Moreover, PSS/PDADMA multilayers constructed in the presence of $\mathrm{NaCl}$ as supporting salt, and thus in conditions of linear growth, seem to be in a glassy state with a $T_{\mathrm{g}}$ on the order of $40^{\circ} \mathrm{C}$, whereas PSS/PAH multilayers have a $T_{\mathrm{g}}$ on the order of $80^{\circ} \mathrm{C}$ (H. Möhwald, personal communication). This may result from a better matching of the distances between the sulfonate groups of PSS and the amine groups of PAH compared to those of PDADMA. It is thus anticipated that the effect of the nature of the salt ions is much reduced for PSS/PAH compared to PSS/PDADMA. A first study by Silva et al. revealed that the nature of the cation of the salt has only a slight effect on the film thickness as measured in the dry state by ellipsometry but that a stronger effect is observed on the electrochemical response of the film in the presence of hexacyanoferrate(III) ions. ${ }^{13}$ This reveals that the film structure is affected by the nature of the salt cation leading to changes in the film resistance and in the diffusion ability of ions such as $\mathrm{Fe}(\mathrm{CN})_{6}{ }^{3-}$.

The goal of this article is to investigate the effect of the nature of the monovalent anions $\left(\mathrm{F}^{-}, \mathrm{Cl}^{-}, \mathrm{NO}_{3}{ }^{-}, \mathrm{ClO}_{4}{ }^{-}\right)$on the buildup of PSS/PAH multilayers and on their properties at controlled $\mathrm{pH}$. We use quartz crystal microbalance (QCM) to investigate the film buildup. This technique allows in situ measurements of the evolution of the film thickness. We use cyclic voltammetry $(\mathrm{CV})$ to get information about the permeability of these films with respect to the multivalent hexacyanoferrate(II) ions $\left(\mathrm{Fe}(\mathrm{CN})_{6}{ }^{4-}\right)$ and ruthenium(II) hexamine ions $\left(\mathrm{Ru}\left(\mathrm{NH}_{3}\right)_{6}{ }^{2+}\right)$ that serve as electroactive probes. $\mathrm{CV}$ will also allow getting information about the presence of excess of fixed charges in the film, a problem longstanding debated. Indeed, first results obtained by Schlenoff et al. ${ }^{14,15}$ suggested that there is almost an exact compensation between the charges brought by the polyanion and those brought by the polycation for strong polyelectrolytes. On the other hand, other results, in particular selective ion transport across multilayers, suggest the presence of fixed charges leading to partial rejection of multivalent ions due to Donnan permse-

(11) Salomäki, M.; Laiho, T.; Kankare, J. Macromolecules 2004, 37, 95859590.

(12) Schmitt, J.; Grünewald, T.; Decher, G.; Pershan, P. S.; Kjaer, K.; Lösche, M. Macromolecules 1993, 26, 7058-7063.

(13) Silva, T. H.; Garcia-Morales, V.; Moura, C.; Manzanares, J. A.; Silva, F. Langmuir 2005, 21, 7461-7467.

(14) Schlenoff, J. B.; Ly, H.; Li, M. J. Am. Chem. Soc. 1998, 120, 7626-7634. (15) Farhat, T. R.; Schlenoff, J. B. J. Am. Chem. Soc. 2003, 125, 4627-4636. lectivity. ${ }^{16-18}$ It must be pointed out that our paper is thus not intended to contribute to a better understanding of the Hofmeister effect but exclusively to the consequences of the anion nature on the PSS/PAH multilayer buildup and structure.

The paper is organized as follows. The following section describes the materials used for the measurements and the methods. The results of both the quartz crystal microbalance, which was used to follow the buildup of PSS/PAH multilayers in different supporting salts, and the voltammetry measurements are shown. The influence of the number of layers and of the anion of the supporting salt on the electrochemical response of $\mathrm{Fe}(\mathrm{CN})_{6}{ }^{4-}$ and $\mathrm{Ru}\left(\mathrm{NH}_{3}\right)_{6}{ }^{2+}$ were studied and discussed in the next section. The manuscript ends with concluding remarks.

\section{Materials and Methods}

Chemicals. Polyethyleneimine (PEI, $M=750000 \mathrm{~g} / \mathrm{mol}$, CAS 18 197-8), poly(sodium 4-styrene sulfonate) (PSS, $M=70000 \mathrm{~g} / \mathrm{mol}$, CAS 24 305-1), and poly(allylamine hydrochloride) (PAH, $M=$ $56000 \mathrm{~g} / \mathrm{mol}$, CAS 28 322-3) were purchased from Aldrich and used as received. Sodium fluoride (Merck), sodium chloride (VWR), sodium nitrate (Sigma-Aldrich), and sodium perchlorate (SigmaAldrich) served as supporting electrolytes $(150 \mathrm{mM}$ in all the cases described below). As far as the anions are concerned, the total ionic strength of the PAH solutions is actually slightly higher (ca. 161 $\mathrm{mM}$ ) due to the contribution of the chloride counterions accompanying the PAH chains. The tris(hydroxymethyl)aminomethane or 2-amino-2-(hydroxymethyl)-propane-1,3-diol $\left(\left(\mathrm{HOCH}_{2}\right)_{3} \mathrm{CNH}_{2}\right.$, usually called Tris), used as a buffer molecule, was purchased from Sigma (CAS 77-86-1). Its concentration was fixed to $5 \mathrm{mM}$ in all the experiments described hereafter. The potassium hexacyanoferrate(II), $\mathrm{K}_{4}\left[\mathrm{Fe}(\mathrm{CN})_{6}\right]$, was supplied by Sigma $(M=422.41 \mathrm{~g} / \mathrm{mol}$, CAS 14459-95-1) and the ruthenium(II) hexaamine chloride, $\mathrm{Ru}\left[\left(\mathrm{NH}_{3}\right)_{6}\right] \mathrm{Cl}_{2}$, by Aldrich $(M=274.16 \mathrm{~g} / \mathrm{mol}$, CAS $203690-1 \mathrm{G})$. All solutions were prepared with ultra pure water (Milli Q Plus system, Millipore, Billerica, MA). The $\mathrm{pH}$ of the four salt solutions, supplemented with $5 \mathrm{mM}$ Tris, was between 9.9 and 10.0. This $\mathrm{pH}$ was reduced to 7.4 using the acid corresponding to the salt (i.e., HF, $\mathrm{HCl}, \mathrm{HNO}_{3}$, or $\mathrm{HClO}_{4}$ ). Caution: hydrofluoric acid is toxic and corrosive; it should be handled with great care. In the four cases, $500-600 \mu \mathrm{L}$ of $1 \mathrm{M}$ acid was added to $140 \mathrm{~mL}$ of the corresponding buffered salt solution to achieve $\mathrm{pH}$ 7.4. The final concentration of $\mathrm{F}^{-}, \mathrm{Cl}^{-}, \mathrm{NO}_{3}{ }^{-}$, and $\mathrm{ClO}_{4}{ }^{-}$ions was thereby slightly increased from $150 \mathrm{mM}$ up to less than $154 \mathrm{mM}$.

Film Preparation. The films were built up by the dipping method ${ }^{19}$ following the usual sequence of substrate dipping into the polyelectrolyte and rinsing solutions. The glassy carbon (GC) electrode (MF 2070, BASI Instruments, Stareton, U.K.), after polishing, was first dipped into the PEI solution $(1 \mathrm{~g} / \mathrm{L})$ for $5 \mathrm{~min}$, followed by a rinsing of $5 \mathrm{~min}$ in the PEI-free solution. The same sequence was used for the PSS (1 g/L) and PAH (1 g/L) alternate depositions. The PSS and PAH adsorptions were continued until the desired number of layers was attained. Then, the film has the structure symbolized by the formula PEI-(PSS/PAH) ${ }_{n}$ (positively charged last layer) or PEI-(PSS/PAH) $)_{n}-\mathrm{PSS}$ (negatively charged last layer), where $n$ is a integer $(n \geq 0)$.

Quartz Crystal Microbalance and Electrochemical Measurements. The quartz crystal microbalance measurements were carried out on the QCM-D301 model from Q-Sense AB (Gothenburg, Sweden) equipped with a quartz crystal coated with $50 \mathrm{~nm}$ of silica. The fundamental oscillation frequency of this quartz oscillator is about $5 \mathrm{MHz}$. This apparatus allows the frequency and dissipation shifts at 5, 15, 25, and $35 \mathrm{MHz}$ to be monitored. Although the PEM film deposition were performed on different substrates, namely silica

(16) Harris, J. J.; Stair, J. L.; Bruening, M. L. Chem. Mater. 2000, 12, 19411946.

(17) Krasemann, L.; Tieke, B. Langmuir 2000, 16, 287-290.

(18) Liu, Y.; Zhao, M.; Bergbreiter, D. E.; Crooks, R. M. J. Am. Chem. Soc. 1997, 119, 8720-8721.

(19) Decher, G. Science 1997, 277, 1232-1237. 


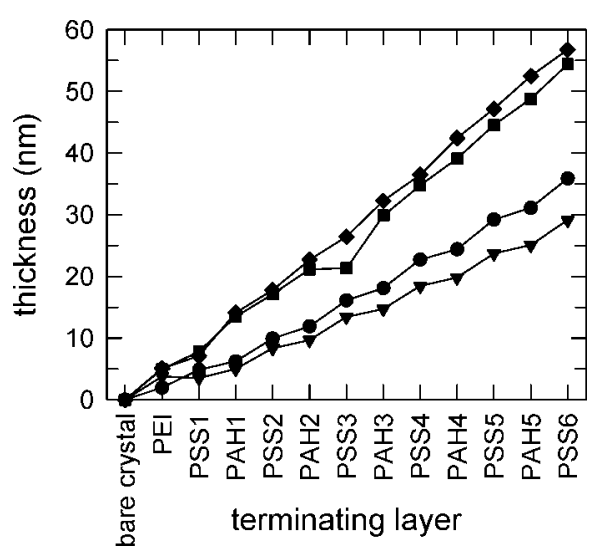

Figure 1. Thickness evolution during the buildup of a PEI-(PSS/ $\mathrm{PAH})_{5}-\mathrm{PSS}$ film in the presence of various anions: $\mathrm{F}^{-}$(circles), $\mathrm{Cl}^{-}$ (triangles), $\mathrm{NO}_{3}{ }^{-}$(squares), $\mathrm{ClO}_{4}{ }^{-}$(diamonds). The thickness is derived from the frequency shift for $v=3$ using Sauerbrey's relation.

and glassy carbon for the QCM and CV experiments, respectively, we assume the film growth to be qualitatively similar. The effect of the initial substrate is expected to be reduced by the adsorption of a PEI layer aimed at priming the deposition of the PEM film.

The electrochemical measurements were performed with a $\mathrm{CHI}$ 604B model (CH Instruments Inc., Austin, TX) and a three-electrode cell where the working electrode was a GC electrode, the reference potential was given by an $\mathrm{Ag} / \mathrm{AgCl}$ electrode, and the counter (or auxiliary) electrode was a platinum wire. The GC disk electrode (diameter $3 \mathrm{~mm}$ ) was polished with $0.05-\mu \mathrm{m}$ alumina powder (Buehler, Lake Bluff, IL) on a polishing microcloth, sonicated, and rinsed with Milli Q water. Before measurement, the solutions were purged with nitrogen for $10 \mathrm{~min}$. During the measurements, a nitrogen layer was maintained above the solution to avoid contamination by the oxygen of air. It is worth noting that, after construction of the film on the electrode, the latter was immersed in the measurement solution, which in all the cases contained the electroactive probe.

\section{Results}

Film Thickness Measured with Quartz Crystal Microbalance. The resonance frequencies and the dissipation factors have been measured during the buildup of the PEI-(PSS/PAH $)_{5}-\mathrm{PSS}$ film for the overtones $v=3,5$, and 7 corresponding to the approximate uncoated crystal frequencies 15,25 , and $35 \mathrm{MHz}$. The reference frequencies and reference dissipations were measured when the crystal was in contact with the buffered saline solutions. The increase of the dissipation due to the progressive deposition of the polyelectrolytes was small in all the cases. On the other hand, the normalized frequency shifts (i.e., the frequency shifts divided by their respective overtone) were practically undistinguishable. Both observations indicate that the films are tightly bound to the surface and behave as solid films. The estimation of the mass deposited per unit area can therefore be based on Sauerbrey's relation..$^{20}$ Furthermore, assuming a specific mass (arbitrarily chosen to be equal to $1 \mathrm{~g} / \mathrm{cm}^{3}$ ) for the polyelectrolyte film allows converting the mass into the thickness of the film. The results are shown in Figure 1.

It appears that the thicknesses corresponding to $\mathrm{F}^{-}$and $\mathrm{Cl}^{-}$ are significantly smaller than those corresponding to films constructed with $\mathrm{NO}_{3}{ }^{-}$and $\mathrm{ClO}_{4}{ }^{-}$as the anions of the supporting electrolyte. It is worth noting that, although the thickness increases more rapidly with $\mathrm{NO}_{3}{ }^{-}$and $\mathrm{ClO}_{4}{ }^{-}$than with $\mathrm{F}^{-}$and $\mathrm{Cl}^{-}$, the buildup regime is still linear, in contrast to the supralinear growth mode observed when PDADMA is used as the polycation and the film constructed in the presence of $\mathrm{Cl}^{-}$or $\mathrm{NO}_{3}^{-}{ }^{-9}$ The QCM

(20) Sauerbrey, G. Z. Phys. 1959, 155, 206-222.

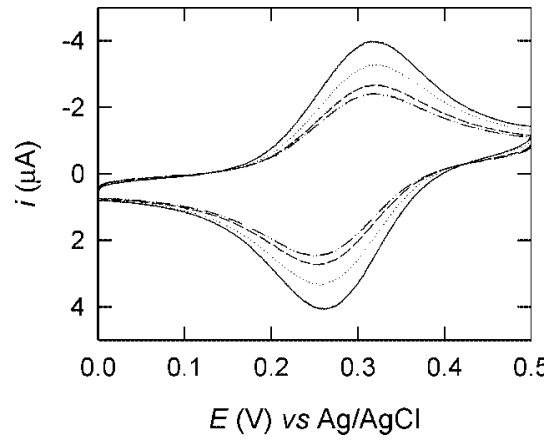

Figure 2. Cyclic voltamograms recorded on a glassy carbon electrode modified by a PEI-(PSS/PAH) $)_{5}-\mathrm{PSS}$ film at $100 \mathrm{mV} / \mathrm{s}$ in the presence of $150 \mathrm{mM} \mathrm{NaNO}_{3}$ as the supporting electrolyte and of $1 \mathrm{mM} \mathrm{Fe}(\mathrm{CN})_{6}{ }^{4-}$ as the redox probe. The perturbation of the films caused by the probing leads to a progressive reduction of the current intensities (the cycles narrow from the first to the fourth passage at $100 \mathrm{mV} / \mathrm{s}$ ).

measurements confirm that the thickness of a PSS/PAH film can be modulated by the choice of the supporting electrolyte anion.

Electrochemistry. PEI-(PSS/PAH $)_{5}-\mathrm{PSS}$ films have been built in the presence of various anions on a GC electrode as described in the subsection "Film Preparation". The capacitive current was measured at various potential sweep speeds $(v=10$, $25,50,75$, and $100 \mathrm{mV} / \mathrm{s}$ ) in the potential domain ranging from $E=0$ up to $E=0.5 \mathrm{~V}$ versus $\mathrm{Ag} / \mathrm{AgCl}$ reference in the presence of either $\mathrm{NaF}$ or $\mathrm{NaCl}$ or $\mathrm{NaNO}_{3}$ or $\mathrm{NaClO}_{4}$ as supporting electrolyte (see Supporting Information).

To probe the film with $\mathrm{Fe}(\mathrm{CN})_{6}{ }^{4-}$, the film was built as described above. After construction on the GC electrode, the electrode was immersed for $1 \mathrm{~h}$ in the same buffered salt solution but supplemented with $1 \mathrm{mM} \mathrm{Fe}(\mathrm{CN})_{6}{ }^{4-}$. After this period of time, the film was submitted, still in the presence of $\mathrm{Fe}(\mathrm{CN})_{6}{ }^{4-}$, to voltammetric cycling at various potential sweep rates. Specifically, 15 cycles (with $E$ ranging from 0 up to $0.5 \mathrm{~V}$ and back to 0 ) were performed successively at $v=100,75,50,25$, and $10 \mathrm{mV} / \mathrm{s}$. This sequence was repeated three times, and the probing was finally ended with a fourth group of 15 cycles at $100 \mathrm{mV} / \mathrm{s}$. For all salts, the four measurements at $100 \mathrm{mV} / \mathrm{s}$, approximately spaced by $1 \mathrm{~h}$, revealed a current intensity decreasing from the 1 st to the 15 th cycle of each passage as well as a global decrease from the 1st passage to the 4th passage at $100 \mathrm{mV} / \mathrm{s}$. Figure 2 shows the 15 th cycle of each of the four passages at $100 \mathrm{mV} / \mathrm{s}$ for the film constructed in $\mathrm{NaNO}_{3}$. Similar results were obtained for the other salts (see Figure S3 of the Supporting Information).

The measurements reported in Figure 2 evidence that the probing alters appreciably the film. The peak intensities decrease regularly as the electrochemical cycles go on. This clearly indicates an evolution of the film structure during the electrochemical probing. A decrease of the intensity occurs while both the potential at the oxidation peak, $E_{\mathrm{p}}^{\mathrm{ox}}$, and the potential at the reduction peak, $E_{\mathrm{p}}^{\mathrm{red}}$, hence also the apparent redox potential, $E_{1 / 2}$ $=1 / 2\left(E_{\mathrm{p}}^{\mathrm{ox}}+E_{\mathrm{p}}^{\text {red }}\right)$, remain unchanged along the cycling process. The same finding is valid for the three other supporting salts considered (Figure S3 of the Supporting Information). To compare the electrochemical response of films constructed in the presence of different ions, it is thus of great importance to compare the anodic and the cathodic currents measured in the same conditions to eliminate as far as possible the biasing effect of repeated cycling.

Before investigating the effect of the nature of the anions during film construction, we studied the effect of the number of layers constituting the film on its electrochemical response. This 

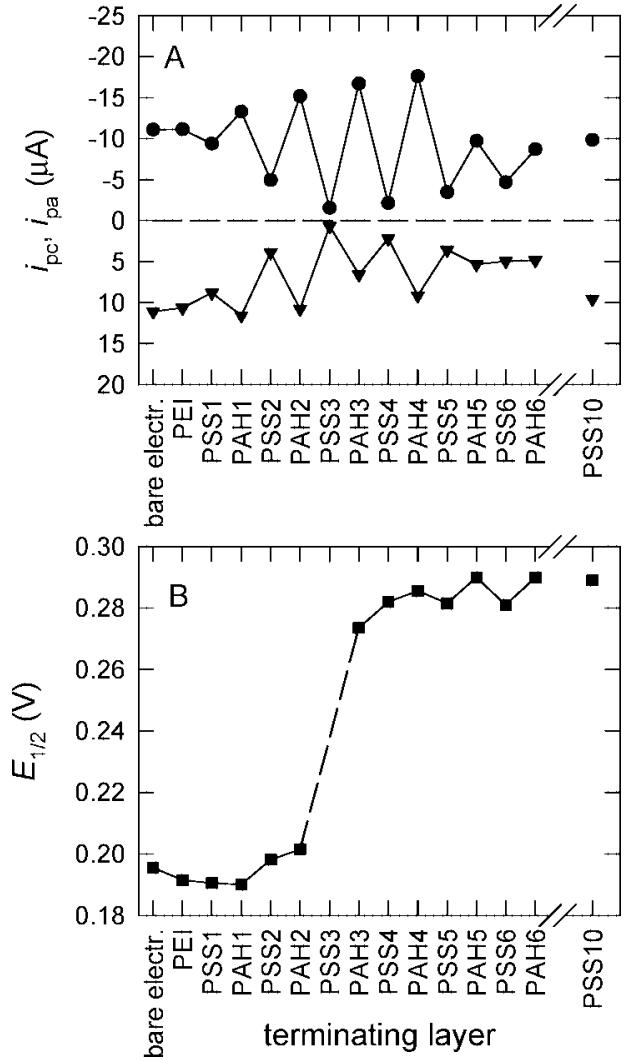

Figure 3. (A) Anodic (circles) and cathodic (triangles) peak currents corresponding to PEI $-(\mathrm{PSS} / \mathrm{PAH})_{n}$ and PEI-(PSS/PAH $)_{n}-\mathrm{PSS}$ films in contact with a buffered $(\mathrm{pH} 7.4) \mathrm{NaNO}_{3}$ solution containing $\mathrm{Fe}(\mathrm{CN})_{6}{ }^{4-}$ at $v=100 \mathrm{mV} / \mathrm{s}$. (B) Apparent redox potential. Since the currents are nearly zero at "PSS3", the peak positions are poorly defined, and therefore, the corresponding data point is missing.

was done only for a given anion, namely $\mathrm{NO}_{3}{ }^{-}$. We thus used $\mathrm{NaNO}_{3}$ as the salt during the multilayer construction. We first used $\mathrm{Fe}(\mathrm{CN})_{6}{ }^{4-}$ as the electrochemical probe. It must be pointed out that each film was built up separately so that the perturbation induced by the probing on one film does not propagate to the next architecture. Figure 3 shows the evolution of both the intensity peaks and that of the apparent redox potential with the number of deposition steps. As can be seen, a transition in the apparent redox potential takes place after "PAH2". The potential jumps from a value close to that corresponding to the bare electrode $(\approx 0.19 \mathrm{~V})$ to a value close to $0.28 \mathrm{~V}$. After the deposition of two bilayers, the potential is always slightly higher for a PAH-terminating film than for a PSS-terminating one, and correlatively, the anodic and cathodic current peaks are much smaller for the films ending with PSS compared to those ending with PAH. The difference between the heights of the anodic and cathodic waves on PAH-terminating films was also observed by others. ${ }^{21}$

We also used a positive electroactive probe, $\mathrm{Ru}\left(\mathrm{NH}_{3}\right)_{6}{ }^{2+}$, to sense the multilayer. The behavior of the film is appreciably different compared to the previous case. This ion is oxidized to $\mathrm{Ru}\left(\mathrm{NH}_{3}\right)_{6}{ }^{3+}$ when the potential of the working electrode is raised from $-0.35 \mathrm{~V}$ up to $0 \mathrm{~V}$ with respect to the reference electrode and then reduced back during the second half of a cycle. Figure 4 , which corresponds to $v=100 \mathrm{mV} / \mathrm{s}$, shows that both the anodic and the cathodic currents decrease in intensity at each PAH deposition, whereas the deposition of PSS has a relatively weaker influence on them. After the deposition of the third PAH

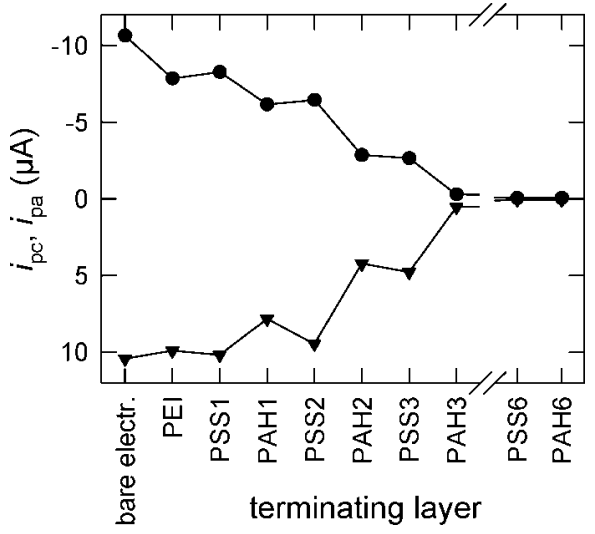

Figure 4. Anodic (circles) and cathodic (triangles) peak currents corresponding to a PEI-(PSS/PAH $)_{n}$ film in contact with a $\mathrm{NaNO}_{3}$ solution containing $\mathrm{Ru}\left(\mathrm{NH}_{3}\right)_{6}{ }^{2+}$ at $v=100 \mathrm{mV} / \mathrm{s}$.

layer ("PAH3"), the currents become practically zero. Note that the blocking of the electrode occurs also with $\mathrm{F}^{-}$and $\mathrm{Cl}^{-}$ions instead of the $\mathrm{NO}_{3}{ }^{-}$ions. The experiment could not be performed with $\mathrm{ClO}_{4}{ }^{-}$ions because the probe precipitates in this solution. Further polyelectrolyte depositions do not change the currents that stay definitively equal to zero within our experimental uncertainty. This observation strongly points to an intrinsic positive charge excess inside the film due to an overcompensation of the negative charges of the PSS chains by the positive charges worn by the PAH chains. ${ }^{22}$ It must also be noticed that, as for $\mathrm{Fe}(\mathrm{CN})_{6}{ }^{4-}$, the apparent redox potential does not vary up to "PAH3". However, in this case, it is no longer defined for thicker films due to the absence of electrochemical response.

The results obtained both on $\mathrm{Fe}(\mathrm{CN})_{6}{ }^{4-}$ and on $\mathrm{Ru}\left(\mathrm{NH}_{3}\right)_{6}{ }^{2+}$ can be explained by a multilayer buildup that takes place in two stages. Up to the buildup of the third PSS/PAH bilayer (i.e., up to "PAH3"), the film only partially covers the surface or presents large holes. This gives acess to the bare electrode to both $\mathrm{Fe}(\mathrm{CN})_{6}{ }^{4-}$ and $\mathrm{Ru}\left(\mathrm{NH}_{3}\right)_{6}{ }^{2+}$ ions from the solution. Once the number of bilayers constituting the $\mathrm{PEI}-(\mathrm{PSS} / \mathrm{PAH})_{n}$ multilayers exceeds 3, the film totally covers the electrode. In the case of $\mathrm{Ru}\left(\mathrm{NH}_{3}\right)_{6}{ }^{2+}$ ions, the multilayer totally prevents access of these ions to the electrode. In order to investigate the influence of the nature of the ions during the film buildup on the electrochemical response, we thus chose to use films made out of 5.5 PSS/PAH bilayers (i.e., PEI-(PSS/PAH $\left.)_{5}-\mathrm{PSS}\right)$. This however allows the study to be only performed with $\mathrm{Fe}(\mathrm{CN})_{6}{ }^{4-}$ ions as the electrochemical probe.

Let us now investigate the effect of the nature of the anions on the electrochemical response of the films. The comparison of the four salts is displayed in Figure 5 where the 15 th voltammetric cycles of the first passage at $v=100 \mathrm{mV} / \mathrm{s}$ are represented. The cycles shown in Figure 5A have been corrected for the respective capacitive currents (see Figure $\mathrm{S} 1$ of the Supporting Information). The influence of the supporting electrolyte appears unambiguously (Figure 5B). More specifically, it follows from these measurements that the anodic and cathodic peak intensities are sensitive to the electrolyte ion and increase along the series $\left(\mathrm{F}^{-}\right.$, $\mathrm{Cl}^{-}, \mathrm{NO}_{3}{ }^{-}, \mathrm{ClO}_{4}{ }^{-}$). This series is in accordance with that observed by Salomäki et al. ${ }^{9}$ as far as $\mathrm{F}^{-}, \mathrm{Cl}^{-}$, and $\mathrm{NO}_{3}{ }^{-}$are concerned and corroborates their expectation on the position of $\mathrm{ClO}_{4}{ }^{-}$. The same sequence of anions was also obtained if it refers to their concentration necessary to make an aqueous salt solution a 

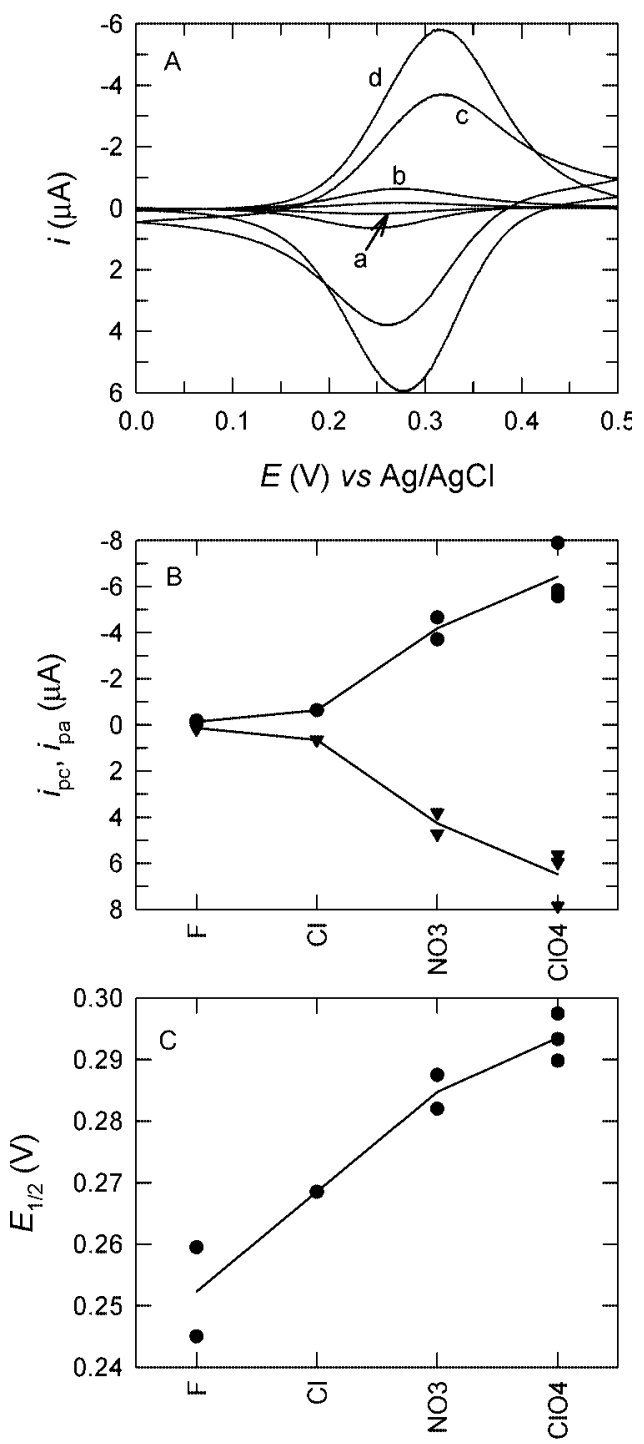

salt anion

Figure 5. (A) Faradic currents corresponding to the 15th cycle of the first passage at $v=100 \mathrm{mV} / \mathrm{s}$ for the four salt anions considered (a: $\mathrm{F}^{-}$; b: $\mathrm{Cl}^{-} ; \mathrm{c}: \mathrm{NO}_{3}{ }^{-} ; \mathrm{d}: \mathrm{ClO}_{4}{ }^{-}$) that served as well for the construction as for the electrochemical characterization, measured for the PEI-(PSS/ $\mathrm{PAH})_{5}-\mathrm{PSS}$ film in contact with $\mathrm{Fe}(\mathrm{CN})_{6}{ }^{4-}$ at $\mathrm{pH}$ 7.4. (B) Anodic (circles) and cathodic (triangles) peak currents. (C) Apparent redox potential. In panels $\mathrm{B}$ and $\mathrm{C}$, the line serves merely to guide the eye.

$\theta$-solvent for cationic polyelectrolytes. ${ }^{23}$ The relatively small difference between the peak current intensities for $\mathrm{F}^{-}$and $\mathrm{Cl}^{-}$ may be related to the weak selectivity of a PSS/PAH film used in nanofiltration aimed at separating $\mathrm{F}^{-}$and $\mathrm{Cl}^{-}$ions in drinking water. ${ }^{24}$ The effect of the nature of the anion manifests also in the apparent redox potential (Figure 5C). This potential is shifted toward positive values when compared to the bare GC electrode $(0.26-0.30 \mathrm{~V}$ compared to $0.19 \mathrm{~V})$, and it depends upon the nature of the anion used for the film construction, more chaotropic anions leading to higher potentials.

To further illustrate the influence of the supporting electrolyte on the response of the redox probe, a PEI-(PSS/PAH $)_{5}-\mathrm{PSS}$ film was constructed in a buffered $\mathrm{NaClO}_{4}$ solution as above.

(23) Burkhardt, C. W.; Parazak, D. P.; McCarthy, K. J.; Jackson, G. J. J. Appl. Polym. Sci. 1986, 32, 4701-4708.

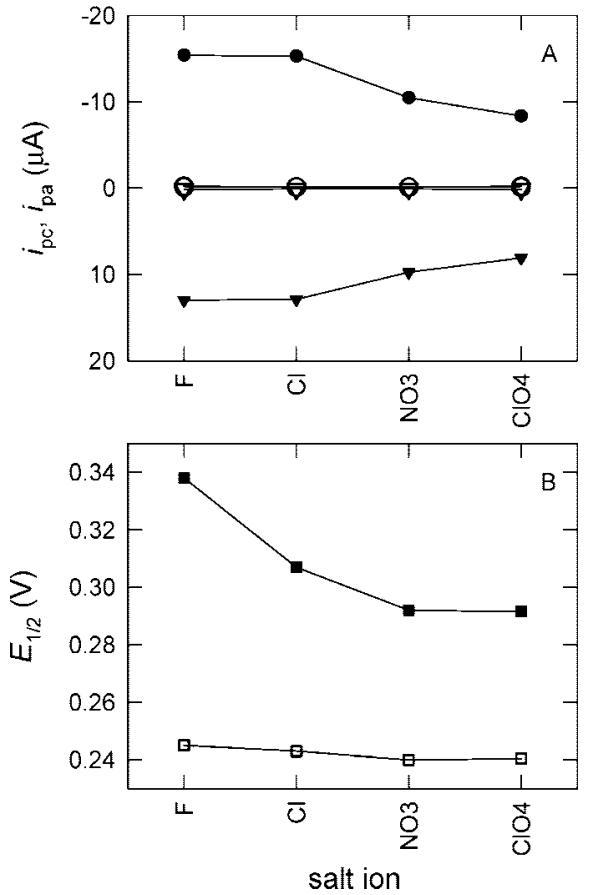

Figure 6. (A) Anodic (circles) and cathodic (triangles) currents measured on PEI-(PSS/PAH) $)_{5}$-PSS films built up in the presence of either $\mathrm{NaClO}_{4}$ (black symbols) or $\mathrm{NaF}$ (white symbols) and probed in contact with a $\mathrm{Fe}(\mathrm{CN})_{6}{ }^{4-}$ solution at $\mathrm{pH} 7.4$ containing the anion indicated on the abscissa scale. (B) Apparent redox potential. $v=100 \mathrm{mV} / \mathrm{s}$.

Then, it was immersed for $1 \mathrm{~h}$ in a buffered salt solution, containing $1 \mathrm{mM} \mathrm{Fe}(\mathrm{CN})_{6}{ }^{4-}$, the supporting salt being either $\mathrm{NaF}$ or $\mathrm{NaCl}$ or $\mathrm{NaNO}_{3}$ or $\mathrm{NaClO}_{4}$. The use of $\mathrm{NaClO}_{4}$ in the measurement step of an experiment was repeated as a control. As can be seen in Figure 6A, the anodic and cathodic currents are sensitive to the salt dissolved in the measuring solution. It may be especially noticed that the $\mathrm{F}^{-}$and $\mathrm{Cl}^{-}$ions do amplify the current intensities by a factor of about 2 when compared to the intensities corresponding to $\mathrm{ClO}_{4}{ }^{-}$. This series of experiments also leads to the ordering of the four anions already observed (i.e., $\mathrm{F}^{-}, \mathrm{Cl}^{-}, \mathrm{NO}_{3}{ }^{-}, \mathrm{ClO}_{4}^{-}$). In Figure $6 \mathrm{~B}$, the corresponding apparent redox potential increases too when the $\mathrm{ClO}_{4}{ }^{-}$solution is replaced by solutions of more cosmotropic anions.

The latter experiments have been repeated with $\mathrm{NaF}$ replacing $\mathrm{NaClO}_{4}$. It is observed (Figure 6A and $\mathrm{B}$ ) in this case that the salt anion in the measuring solution has a significant incidence neither on the current intensities nor on the apparent redox potential.

\section{Discussion and Proposed Model}

In the previous analysis of the experimental data, we focused on both the anodic and cathodic peak intensities and on the apparent redox potential. The peak intensities are sensitive to the local concentration of electroactive species, $\mathrm{Fe}(\mathrm{CN})_{6}{ }^{4-}$ ions in our case, at the surface of the GC electrode. This local concentration, during a voltammetry cycle, depends upon the concentration of $\mathrm{Fe}(\mathrm{CN})_{6}{ }^{4-}$ ions in the film and upon their diffusion ability through the multilayer. The shift of the apparent redox potential, when compared to the bare electrode, is sensitive to the Donnan potential inside the multilayer. ${ }^{25}$ The Donnan potential is mainly due to "fixed" charges in the film. They originate from the imbalance between the charges carried by the polyanions (PSS) and those carried by the polycations (PAH). 
In this case, the film electroneutrality requires charge compensation through extrinsic charges brought by the ions in equilibrium with the solution. Contributions of nonelectrostatic interactions between the electroactive ions and the polyelectrolytes can also contribute to the shift of the apparent redox potential. The fact that, for a (PSS/PAH) multilayer constituted of more than three bilayers, the shift of the apparent redox potential is positive indicates that there is a positive Donnan potential in the film. This positive Donnan potential must originate from an excess of "fixed" positive charges and explains the reason why, after the deposition of more than three bilayers, the multilayer becomes impermeable toward $\mathrm{Ru}\left(\mathrm{NH}_{3}\right)_{6}{ }^{2+}$ ions. This observation is in agreement with the AFM measurements performed by Lavalle et al. on the same polyelectrolyte system, in the same salt and $\mathrm{pH}$ conditions, that revealed full coverage of a substrate after about three pairs of layers. $^{26}$

The excess of positive charges in the PSS/PAH multilayer may be due to a strong interaction between the anions and the $\mathrm{NH}_{3}{ }^{+}$groups worn by the PAH chains. This strong interaction would reduce the effective charge of the PAH chains as was previously demonstrated in the case of $\mathrm{SCN}^{-}$anions ${ }^{27}$ and thus the number of $\mathrm{NH}_{3}{ }^{+}$groups directly involved in $\mathrm{NH}_{3}{ }^{+} / \mathrm{SO}_{3}{ }^{-}$ electrostatic interactions between the PSS and the PAH chains. These $\mathrm{NH}_{3}{ }^{+}$groups, partially neutralized by the counteranions, then form the excess of "fixed" positive charges. Thus, the stronger the interaction between the anions and the PAH chains, the higher should be the excess of positive charges in the film (i.e., charges that do not contribute to the intrinsic compensation of the negative charges of the PSS chains) and the more positive should be the Donnan potential. This is indeed observed in Figure 5 where the apparent redox potential increases as the anion becomes more chaotropic. This partial neutralization of the positive charges along the PAH chains by the anions from the solution could also explain why the thickness of the multilayer is higher with anions that bound more strongly to PAH. As shown by Itaya and Ochiai, a stronger bonding to PAH is observed when the chaotropic nature of the anion increases. ${ }^{28}$ These authors explain this stronger binding of chaotropic anions to $\mathrm{PAH}$ by a hydration effect. However, this binding can have different origins such as a low dielectric constant near an interface (here the polyelectrolyte), hydrophobic effects, or polarizability of the anions as discussed in details by Jungwirth and Winter. ${ }^{29}$ This is however out of the scope of the present study. Therefore, there is an anion specific counterion condensation ${ }^{30}$ on the $\mathrm{PAH}$ chain that helps to change the Donnan potential of the film. Such examples of specific counterion condensation are widespread in the literature and can even lead to a charge inversion of the polyanions in the presence of monovalent counter-ions, as predicted theoretically and measured experimentally. ${ }^{31,27}$ Indeed, a partial neutralization of the $\mathrm{NH}_{3}{ }^{+}$groups on $\mathrm{PAH}$ leads to chains that interact with an interface in more loopy conformations. This explanation, already advanced by Salomäki et al., ${ }^{9}$ is confirmed here by the electrochemical experiments. A picture of the PSS/PAH buildup is summarized schematically in Figure 7. The stronger interaction of chaotropic anions with PAH chains explains also the higher peak intensities observed with the $\mathrm{Fe}(\mathrm{CN})_{6}{ }^{4-}$ probe when one uses more chaotropic ions for the film buildup. This higher peak intensity has two origins: (i) a

(26) Lavalle, P.; Gergely, C.; Cuisinier, F. J. G.; Decher, G.; Schaaf, P.; Voegel, J.-C.; Picart, C. Macromolecules 2002, 35, 4458-4465.

(27) Ball, V.; Voegel, J.-C.; Schaaf, P. Langmuir 2005, 21, 4129-4137.

(28) Itaya, T.; Ochiai, H. J. Polym. Sci. Pt. B-Polym. Phys. 1992, 30, 587-590.

(29) Jungwirth, P.; Winter, B. Annu. Rev. Phys. Chem. 2008, 59, 343-366.

(30) Manning, G. S. J. Chem. Phys. 1969, 51, 924-933.

(31) Nguyen, T. T.; Grosberg, A. Y.; Shklovskii, B. I. Phys. Rev. Lett. 2000, $85,1568-1571$.

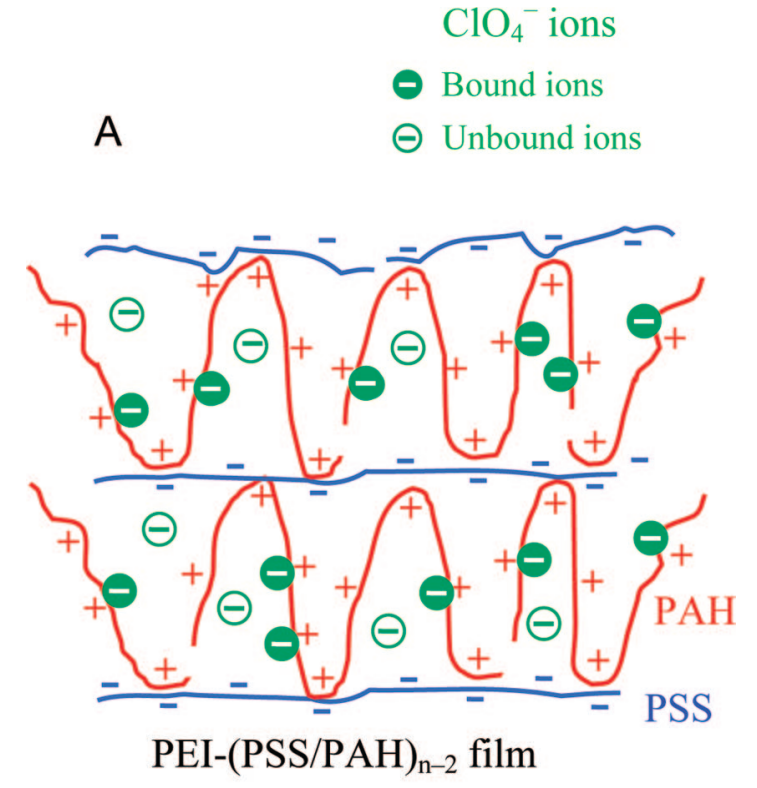

substrate

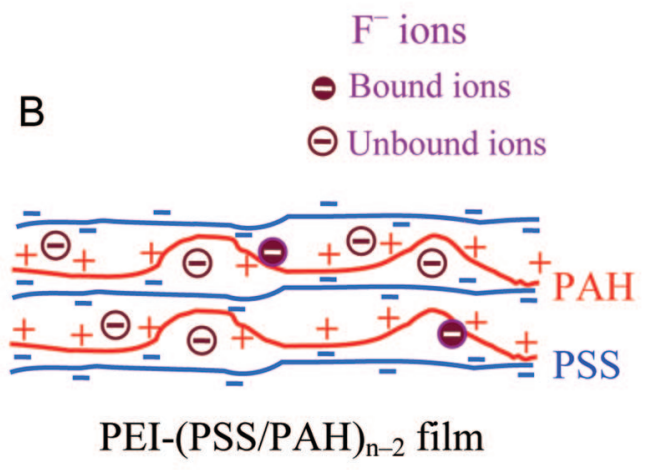

substrate

Figure 7. Schematic representation of the buildup of a PEM film from PSS and PAH solutions. (A) The supporting salt anions are chaotropic; their interaction with the polycation is strong and thereby partially neutralizes its positive charges. This leads to a loopy configuration. (B) The supporting salt anions are cosmotropic; their interaction with the polycation is less strong. The charge density along the chain is therefore higher, which leads to a straighter configuration. This then leads also to better intrinsic charge compensation in the film.

more positive Donnan potential leads to a higher concentration of multivalent anions in the film; (ii) a more loopy conformation leads to less dense films and thus to a higher mobility and diffusion ability of the $\mathrm{Fe}(\mathrm{CN})_{6}{ }^{4-}$ ions and electrolyte ions through the films. Both factors induce higher anodic and cathodic peak intensity, as observed experimentally (Figure 5).

Let us now explain the behavior of the PEM film built in the presence of $\mathrm{ClO}_{4}{ }^{-}$anions and afterward brought into contact with solutions with different anions. When $\mathrm{ClO}_{4}^{-}$ions are replaced by more cosmotropic anions, an increase of the peak intensities and of the Donnan potential is observed. This behavior is opposite to that discussed previously. In fact, this can be explained as follows. Once a multilayer is built with $\mathrm{ClO}_{4}{ }^{-}$anions, some of the $\mathrm{NH}_{3}{ }^{+}$groups that are not compensated by the $\mathrm{SO}_{3}{ }^{-}$groups of PSS are in strong interaction with the $\mathrm{ClO}_{4}{ }^{-}$anions. These groups thus appear as neutral, even for the Donnan potential. When the solution containing $\mathrm{ClO}_{4}{ }^{-}$anions is replaced by a solution containing more cosmotropic anions like $\mathrm{Cl}^{-}$or $\mathrm{F}^{-}$ 

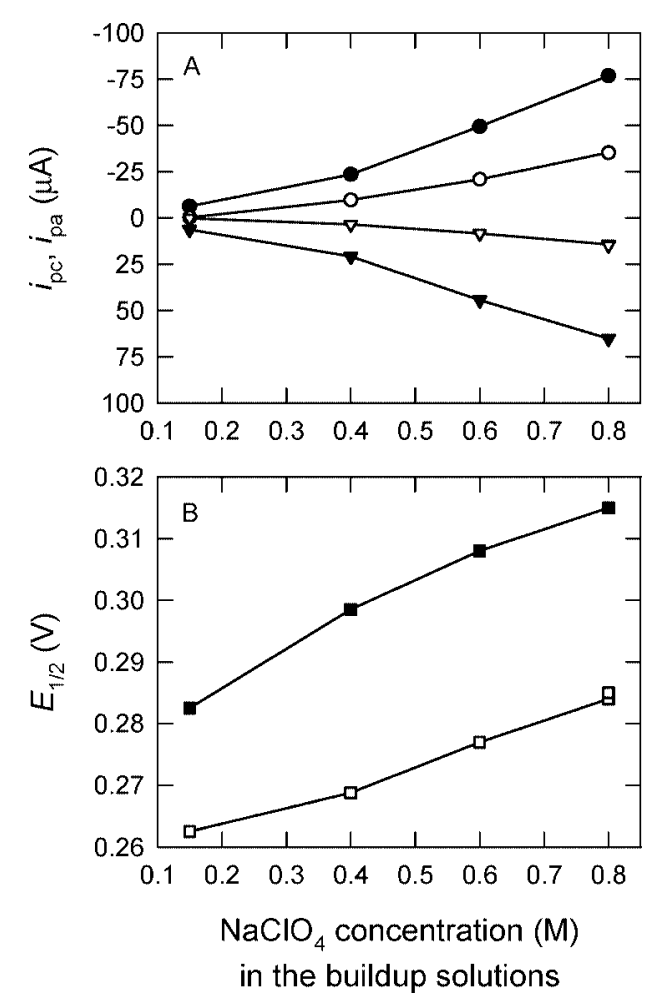

Figure 8. (A) Anodic (circles) and cathodic (triangles) currents measured on PEI-(PSS/PAH $)_{5}-\mathrm{PSS}$ films built up in the presence of $\mathrm{NaClO}_{4}$ at various concentrations and probed in contact with a $\mathrm{Fe}(\mathrm{CN})_{6}{ }^{4-}$ solution at $\mathrm{pH} 7.4$ containing $\mathrm{NaClO}_{4}$ at either $0.15 \mathrm{M}$ (black symbols) or 0.8 $\mathrm{M}$ (white symbols). (B) Apparent redox potentials. $v=100 \mathrm{mV} / \mathrm{s}$.

anions, these anions also replace the $\mathrm{ClO}_{4}{ }^{-}$anions inside the film. Because the $\mathrm{Cl}^{-}$or $\mathrm{F}^{-}$anions bind less strongly to $\mathrm{PAH}$ than the $\mathrm{ClO}_{4}{ }^{-}$anions, less $\mathrm{NH}_{3}{ }^{+}$groups appear as neutral and the density of "fixed" positive charges increases. Moreover, because less $\mathrm{Cl}^{-}$or $\mathrm{F}^{-}$ions are bound to the PAH chains, more of them are free and cause an increase in the osmotic pressure. In addition, the higher density of "free" $\mathrm{NH}_{3}{ }^{+}$groups also induces a stronger electrostatic repulsion between the PAH chains inside the film. All these effects concur toward the swelling of the film. This swelling in turn induces a higher mobility and diffusion ability of the $\mathrm{Fe}(\mathrm{CN})_{6}{ }^{4-}$ ions and the electrolyte ions and thus higher anodic and cathodic peak intensities. On the other hand, when the multilayer is constructed in the presence of cosmotropic anions such as $\mathrm{F}^{-}$ions and when the solution is replaced by a solution containing chaotropic anions such as $\mathrm{ClO}_{4}^{-}$ions, the opposite behavior is a priori expected. In fact, a slight decrease of the Donnan potential is observed when the $\mathrm{F}^{-}$solution is replaced by solutions of more chaotropic anions. This small drop of the potential is due to the fact that, for a film built with $\mathrm{F}^{-}$ anions, the charge compensation inside the film is much more of intrinsic nature so that the density of "free" $\mathrm{NH}_{3}{ }^{+}$groups is much smaller. The consequences of the replacement of the $\mathrm{F}^{-}$ anions by more chaotropic ones can thus only be small.

To further check the validity of the model, PEI-(PSS/ $\mathrm{PAH})_{5}-\mathrm{PSS}$ films have been constructed in the presence of $\mathrm{ClO}_{4}{ }^{-}$ ions at $\mathrm{pH} 7.4$ at various salt concentrations: $0.15,0.4,0.6$, and $0.8 \mathrm{M}$. The peak currents and the apparent redox potential were then measured at either 0.15 or $0.8 \mathrm{M}$ (Figure 8 ). When the measurements are performed in the presence of $\mathrm{ClO}_{4}{ }^{-}$ions at $0.15 \mathrm{M}$, the film either keeps most of its $\mathrm{ClO}_{4}{ }^{-}$ions when constructed at $0.15 \mathrm{M}$ or loses part of these ions when constructed at higher anion concentrations through an exchange between $\mathrm{ClO}_{4}{ }^{-}$and $\mathrm{Fe}(\mathrm{CN})_{6}{ }^{4-}$ anions to preserve electroneutrality. This

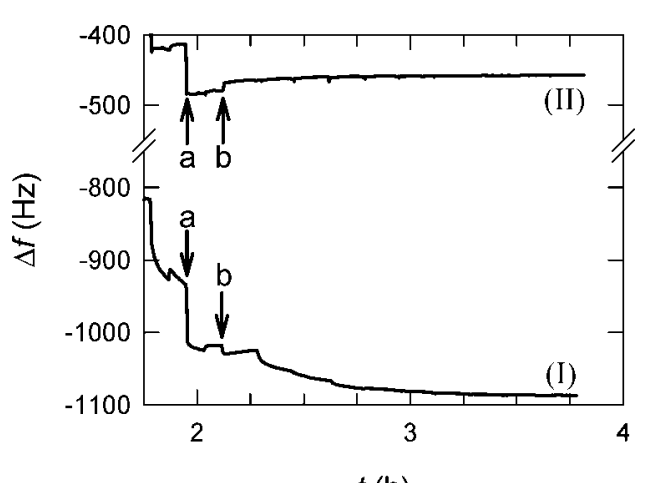

$t$ (h)

Figure 9. Time evolution of $\mathrm{PEI}-(\mathrm{PSS} / \mathrm{PAH})_{5}-\mathrm{PSS}$ film after its construction followed by QCM. The resonance frequency shifts correspond to the third overtone $(\approx 15 \mathrm{MHz})$ of the quartz crystal. Buildup in the presence of $\mathrm{ClO}_{4}{ }^{-}$ions followed by contact with $\mathrm{F}^{-}$ions (I) and buildup in the presence of $\mathrm{F}^{-}$ions followed by contact with $\mathrm{ClO}_{4}^{-}$ions (II); the arrows labeled "a" indicate the injection of the last PSS solution ("PSS6"), and the arrows labeled "b" indicate the time from which the respective salt solutions were injected.

influx, and so the peak current intensities (Figure 8A, black symbols), increases as the difference between the buildup concentration and the measurement concentration increases. Moreover, because of this process, more $\mathrm{NH}_{3}{ }^{+}$groups of the PAH from the multilayer become unscreened and thus act as "fixed" positive charges within the film. Thus, one anticipates an increase of the Donnan potential with the salt concentration difference between buildup and measurement solutions as it is observed experimentally (Figure 8B, black symbols).

Let us now turn to the case where the measurements are performed at $0.8 \mathrm{M}$ instead of $0.15 \mathrm{M} \mathrm{ClO}_{4}{ }^{-}$. In this case, the amount of $\mathrm{Fe}(\mathrm{CN})_{6}{ }^{4-}$ anions replacing $\mathrm{ClO}_{4}{ }^{-}$anions in the film must be much smaller. This leads to a decrease of the peak intensity when compared to experiments performed at $0.15 \mathrm{M}$ (Figure 8A, white symbols). Moreover, because the amount of $\mathrm{ClO}_{4}{ }^{-}$ions that leave the film is reduced compared to the case of measurements performed at $0.15 \mathrm{M}$, the Donnan potential should also be reduced. This again is observed experimentally (Figure 8B, white symbols).

The model described above predicts the swelling of a film constructed in the presence of chaotropic anions (e.g., $\mathrm{ClO}_{4}{ }^{-}$) then exposed to a solution of more cosmotropic anions such as $\mathrm{F}^{-}$(case I). On the opposite, it predicts that a film constructed in the presence of $\mathrm{F}^{-}$ions and further brought in contact with $\mathrm{ClO}_{4}{ }^{-}$ions should shrink (case II). The shift of the resonance frequency corresponding to the third harmonics of the quartz crystal is shown in Figure 9 . In case I, $\Delta f$ falls off by about 60 $\mathrm{Hz}$ after injection of the $\mathrm{F}^{-}$solution. If $\mathrm{C} \mathrm{ClO}_{4}{ }^{-}$solution is replaced by an $\mathrm{F}^{-}$solution on a bare crystal, the drop of frequency is only about $12 \mathrm{~Hz}$. Therefore, the effect observed in Figure 9 corroborates the prediction of a swelling. In case II, $\Delta f$ increases by about $20 \mathrm{~Hz}$ after injection of the $\mathrm{ClO}_{4}{ }^{-}$solution. If a $\mathrm{F}^{-}$ solution is replaced by a $\mathrm{ClO}_{4}{ }^{-}$solution on a bare crystal, the increase of frequency is about $12 \mathrm{~Hz}$. Therefore, the effect observed in Figure 9 exceeds only slightly the effect due to the passage from one electrolyte to the other; nonetheless, it suggests some shrinkage of the film as expected.

Further, if our analysis is correct, then, by keeping the nature of the anion and by reducing or increasing the density of extrinsically compensated $\mathrm{NH}_{3}{ }^{+}$groups compared to the total number of amine groups (whether protonated or not), both the apparent redox potential and the peak current intensities should be reduced or increased, respectively. This can be done by 

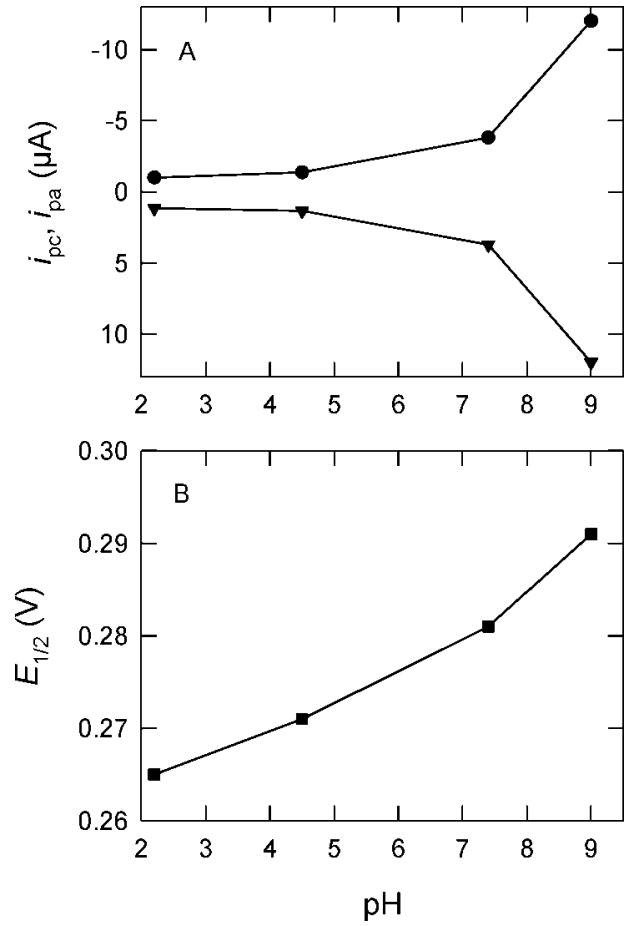

Figure 10. (A) Anodic (circles) and cathodic (triangles) currents measured at $\mathrm{pH} 7.4$ on PEI-(PSS/PAH $)_{5}-\mathrm{PSS}$ films in the presence of $\mathrm{Fe}(\mathrm{CN})_{6}{ }^{4-}$ as a function of the buildup $\mathrm{pH}$ in $\mathrm{NaNO}_{3}$. (B) Apparent redox potential. $v=100 \mathrm{mV} / \mathrm{s}$.

constructing the multilayer at different pHs. We have thus constructed PEI-(PSS/PAH $)_{5}-\mathrm{PSS}$ films in the presence of $\mathrm{NaNO}_{3}$ at $\mathrm{pH}$ 9.0, $\mathrm{pH} 4.5$, and $\mathrm{pH} 2.2$ instead of $\mathrm{pH}$ 7.4. The $\mathrm{CV}$ was however performed at $\mathrm{pH} 7.4$ as above. At the sweep speed of $100 \mathrm{mV} / \mathrm{s}$, we observed an increase in the current intensities from about $3.8 \mu \mathrm{A}$ up to $12.0 \mu \mathrm{A}$ when the film was constructed at $\mathrm{pH} 9.0$ instead of $\mathrm{pH} 7.4$ and a decrease of the intensity from $3.8 \mu \mathrm{A}$ to $1.4 \mu \mathrm{A}$ and $1 \mu \mathrm{A}$ when the multilayer was constructed at $\mathrm{pH} 4.5$ and 2.2 instead of $\mathrm{pH} 7.4$, respectively (Figure 10A). Similarly, we observe an increase in the apparent redox potential when the film is constructed at $\mathrm{pH} 9.0$ and a decrease of $E_{1 / 2}$ when it is constructed at $\mathrm{pH} 4.5$ and 2.2 (Figure 10B). These results are in accordance with our model given above. Indeed, as the $\mathrm{pH}$ increases, more $\mathrm{NH}_{3}{ }^{+}$groups deprotonate to become neutral $\mathrm{NH}_{2}$ groups. For instance, at $\mathrm{pH} 7.4,93 \%$ of the amine groups are protonated whereas only $24 \%$ of them are protonated at $\mathrm{pH} 9.0$ assuming a $\mathrm{p} K_{\mathrm{a}}$ value of 8.5 for $\mathrm{PAH}$. Therefore, during the film buildup at $\mathrm{pH} 9.0$, fewer amine groups interact with the negative $\mathrm{SO}_{3}{ }^{-}$groups located on the PSS chains compared to $\mathrm{pH}$ 7.4. Once the film is constructed and then exposed to a solution at lower $\mathrm{pH}(\mathrm{pH} 7.4)$, the $\mathrm{NH}_{2}$ groups tend to protonate leading to a higher density of positive "fixed" charges inside the film. This leads to a higher Donnan potential for the film constructed at $\mathrm{pH}$ 9.0. Moreover, the protonation of the $\mathrm{NH}_{2}$ groups, when the $\mathrm{pH}$ is reduced from 9.0 during the construction to 7.4 for the measurements, induces a repulsion between the $\mathrm{PAH}$ chains in the film and an increase of the $\mathrm{NO}_{3}{ }^{-}$ion concentration in the film. These effects lead together to the swelling of the film, the increase of the diffusion ability of the ions and thus also to the increase of the anodic and cathodic peak intensities. Qualitatively, the reversed results are observed when the film is built up at $\mathrm{pH} 4.5$ or 2.2 and then again probed at $\mathrm{pH}$ 7.4. These results thus fully support our model.
Our model also explains why the apparent redox potential remains constant during the film restructuring that occurs along successive voltammetry cycles. Indeed, the apparent redox potential shift with respect to the bare electrode corresponds mainly to the Donnan potential. This potential is however directly related to the "fixed" charges in the film that correspond to the excess charges worn by the PAH chains with respect to PSS. As the different cycles take place without the presence of polyelectrolytes in the solution, the charge imbalance in the multilayer can thus not be changed. This implies that the Donnan potential remains constant during cycling, as it is observed.

\section{Conclusions}

In this article, we investigated the effect of the nature of the anions of the supporting electrolyte on the buildup and the permeability to redox species of PSS/PAH multilayers. We confirm a general result observed on other polyelectrolyte multilayers, namely that more chaotropic anions lead to thicker films. We use cyclic voltammetry to sense the structure of the multilayers. All our electrochemical results can be explained by a stronger binding of chaotropic anions to PAH compared to more cosmotropic ones. As far as the multilayer buildup is concerned, such a strong binding is equivalent to a deprotonation of PAH as it is observed when the film is constructed at a higher $\mathrm{pH}$. The strong binding of chaotropic anions with $\mathrm{NH}_{3}{ }^{+}$groups of PAH leads to an adsorption of PAH chains in more loopy conformations as already suggested by Salomäki et al. ${ }^{9}$ It also leads to an excess of "fixed" positive charges in the film and thus to a positive Donnan potential. This potential is the more positive that the supporting anion during film buildup is more chaotropic. Finally, this model also predicts that a film constructed with chaotropic anions in the supporting electrolyte swells and becomes accordingly more permeable to the electroactive probe when the solution is replaced by a solution containing more cosmotropic anions, a prediction that is also confirmed experimentally. We thus arrive to a coherent explanation of the effect of the nature of the anions of the supporting electrolyte on the PEM films.

In the parts of this study that deal with the anion effect, we followed the "one-factor-at-a-time" principle as we changed only the anion of the electrolyte from one experiment to the next. However, this principle appears to be in debate especially as far as the effect of ions is concerned in the context of Hofmeister series. ${ }^{32}$ Indeed, in each case, we adjusted the $\mathrm{pH}$ to 7.4, whereas it should be an independent variable, and in addition, we used a buffer molecule to maintain this $\mathrm{pH}$ value during the measurements. Thus, the intrinsic influence of the anions tested may be partly blurred. Nevertheless, at least from a practical point of view, the present work reveals that changing the anion of the polyelectrolyte buildup solutions permits both the thickness and the permeability of the films to be varied in a predictable way while keeping the same $\mathrm{pH}$ of all the solutions.

Acknowledgment. A.E.E.H. thanks the Ministère de l'Enseignement Supérieur et de la Recherche for financial support.

Supporting Information Available: Capacitive currents measured on a PEI-(PSS/PAH $)_{5}-$ PSS film in the presence of fluoride, chloride, nitrate, and perchlorate ions. Corresponding cyclic voltamograms in the presence of $\mathrm{Fe}(\mathrm{CN})_{6}{ }^{4-}$. This material is available free of charge via the Internet at http://pubs.acs.org.

\section{LA803534Y}

(32) Evens, T.; Niedz, R. Scholarly Res. Exch. In press, doi:10.3814/2008/ 818461. 